\title{
ARTICLE
}

\section{Chronotype and cellular circadian rhythms predict the clinical response to lithium maintenance treatment in patients with bipolar disorder}

\author{
Michael J. McCarthy $\mathbb{D}^{1,2}$, Heather Wei ${ }^{2}$, Caroline M. Nievergelt $\mathbb{D}^{1}$, Andrea Stautland ${ }^{3}$, Adam X. Maihofer (D) ${ }^{1}$, David K. Welsh ${ }^{1,2}$, \\ Paul Shilling ${ }^{1}$, Martin Alda ${ }^{4}$, Ney Alliey-Rodriguez $\mathbb{D}^{5}$, Amit Anand ${ }^{6}$, Ole A. Andreasson ${ }^{7}$, Yokesh Balaraman ${ }^{6}$, Wade H. Berrettini ${ }^{8}$, \\ Holli Bertram ${ }^{9}$, Kristen J. Brennand (D ${ }^{10}$, Joseph R. Calabrese ${ }^{11}$, Cynthia V. Calkin ${ }^{4}$, Ana Claasen (D ${ }^{12}$, Clara Conroy ${ }^{11}$, William H. Coryell ${ }^{13}$, \\ David W. Craig ${ }^{12}$, Nicole D’Arcangelo ${ }^{11}$, Anna Demodena ${ }^{2}$, Srdjan Djurovic (D) ${ }^{7}$ Scott Feeder ${ }^{14}$, Carrie Fisher ${ }^{6}$, Nicole Frazier ${ }^{9}$, \\ Mark A. Frye ${ }^{14}$, Fred H. Gage $\mathbb{i D}^{15}$, Keming Gao ${ }^{11}$, Julie Garnham ${ }^{4}$, Elliot S. Gershon (D) $^{5}$, Kara Glazer ${ }^{16}$, Fernando Goes ${ }^{16}$, Toyomi Goto ${ }^{11}$, \\ Gloria Harrington ${ }^{9}$, Petter Jakobsen ${ }^{17}$, Masoud Kamali ${ }^{9}$, Elizabeth Karberg ${ }^{11}$, Marisa Kelly (iD 9 , Susan G. Leckband ${ }^{2}$, Falk Lohoff ${ }^{8}$, \\ Melvin G. McInnis $\mathbb{D}^{9}$, Francis Mondimore ${ }^{16}$, Gunnar Morken $\mathbb{D}^{18}{ }^{18}$, John I. Nurnberger ${ }^{6}$, Sarah Obral ${ }^{11}$, Ketil J. Oedegaard ${ }^{3,17}$, \\ Abigail Ortiz $\mathbb{I D}^{19}$, Megan Ritchey ${ }^{16}$, Kelly Ryan ${ }^{9}$, Martha Schinagle $^{11}$, Helle Schoeyen ${ }^{3}$, Candice Schwebel ${ }^{8}$, Martha Shaw ${ }^{5}$, \\ Tatyana Shekhtman ${ }^{1,2}$, Claire Slaney ${ }^{4}$, Emma Stapp $\mathbb{D}^{16}$, Szabolcs Szelinger ${ }^{12}$, Bruce Tarwater ${ }^{12}$, Peter P. Zandi ${ }^{16}$ and John R. Kelsoe ${ }^{1,2}$
}

Bipolar disorder (BD) is a serious mood disorder associated with circadian rhythm abnormalities. Risk for BD is genetically encoded and overlaps with systems that maintain circadian rhythms. Lithium is an effective mood stabilizer treatment for BD, but only a minority of patients fully respond to monotherapy. Presently, we hypothesized that lithium-responsive BD patients (Li-R) would show characteristic differences in chronotype and cellular circadian rhythms compared to lithium non-responders (Li-NR). Selecting patients from a prospective, multi-center, clinical trial of lithium monotherapy, we examined morning vs. evening preference (chronotype) as a dimension of circadian rhythm function in $193 \mathrm{Li}-\mathrm{R}$ and Li-NR BD patients. From a subset of 59 patient donors, we measured circadian rhythms in skin fibroblasts longitudinally over 5 days using a bioluminescent reporter (Per2-luc). We then estimated circadian rhythm parameters (amplitude, period, phase) and the pharmacological effects of lithium on rhythms in cells from Li-R and Li-NR donors. Compared to Li-NRs, Li-Rs showed a difference in chronotype, with higher levels of morningness. Evening chronotype was associated with increased mood symptoms at baseline, including depression, mania, and insomnia. Cells from Li-Rs were more likely to exhibit a short circadian period, a linear relationship between period and phase, and period shortening effects of lithium. Common genetic variation in the $\mathrm{IP}_{3}$ signaling pathway may account for some of the individual differences in the effects of lithium on cellular rhythms. We conclude that circadian rhythms may influence response to lithium in maintenance treatment of $\mathrm{BD}$.

Neuropsychopharmacology (2019) 44:620-628; https://doi.org/10.1038/s41386-018-0273-8

\section{INTRODUCTION}

Bipolar disorder (BD) is a disabling psychiatric disorder that affects $1-2 \%$ of the population $[1,2]$. BD is defined by the presence of recurrent depressive and manic/hypomanic episodes, as well as disruptions in rhythmic behaviors. For instance, depressive episodes are characterized by changes in sleep, daytime energy, motivation, and appetite; while mania is characterized by decreased need for sleep and increased nocturnal activity. Even during euthymic periods, BD patients commonly show late onset of morning activity, intrusions of sleep during the day, a preference for evening activity, changes in hormonal rhythms and abnormal temperature cycles indicative of circadian rhythm abnormalities and consistent with delayed phase and/or long period [3-10].

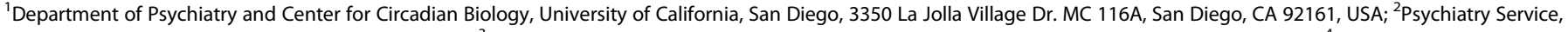

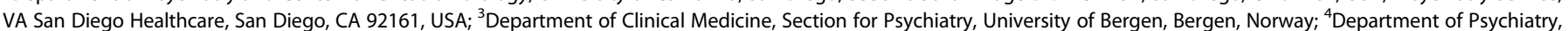

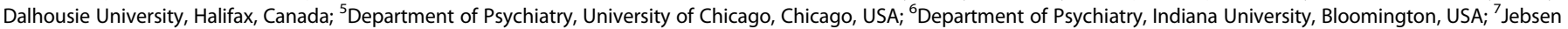

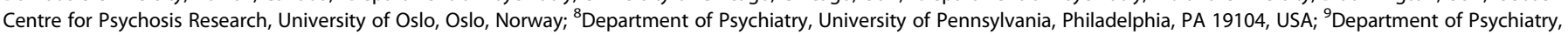

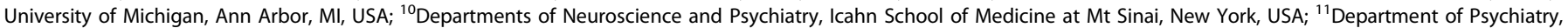

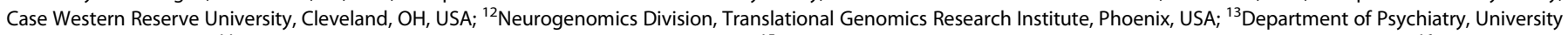

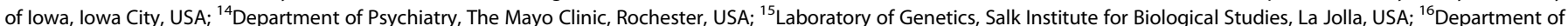

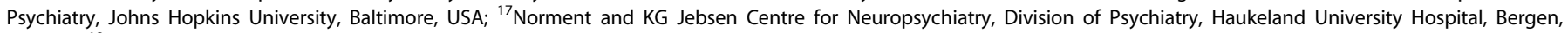

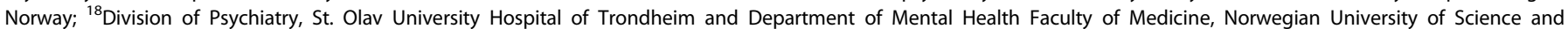
Technology, Trondheim, Norway and ${ }^{19}$ Department of Psychiatry, University of Ottawa, Ottawa, ON, Canada Correspondence: Michael J. McCarthy (mmccarthy@ucsd.edu)
} 
Circadian rhythms are maintained in a cell autonomous fashion by a molecular feedback loop comprised of $\sim 20$ "clock genes" [11]. The essential elements of the loop include the CLOCK/BMAL1 protein complex that drives expression of PER $1 / 2 / 3$ and CRY1/2. Through negative feedback of BMAL1/ CLOCK, PER and CRY inhibit their own expression over $\sim 24 \mathrm{~h}$ cycles leading to rhythms in gene expression. In animals, disruption of clock genes has been used to model mania [12] and depression [13], and clock gene mutants show abnormal responses to lithium in animal models of mania [14]. However, these animal models do not address some core features of BD including grandiosity, speech abnormalities and episodic alternations of mood. In humans, post mortem studies show the loss of circadian rhythms across several brain regions in patients with mood disorders [15]. Moreover, variation in clock genes has been linked to morning/evening preference ("chronotype") both in the general population [16] and in circadian rhythm disorders $[17,18]$. Since the circadian clock is cell autonomous, it can be studied in peripheral cell cultures from humans using bioluminescent reporters. Using this approach, we have shown previously that compared to controls, circadian period is typically longer in cells from BD patients, consistent with the behavioral data observed in human subjects indicating a predisposition for evening activity in BD [19].

Mood stabilizer medications positively impact the course of BD. However, many BD patients fail to respond adequately to pharmacotherapy [20]. Lithium is the best studied, and arguably most effective mood stabilizer, with efficacy in depression, mania and maintenance. However, only about $30 \%$ of patients with $B D$ respond fully to lithium [21]. Like the risk for $B D$ which is largely genetic [22], lithium response is heritable [23], and influenced by genetic variation [24]. Recent genome-wide association studies have identified loci that predict lithium response [25], but the biological processes governed by these genes remain uncharacterized.

Previous preclinical studies have focused on lithium's ability to inhibit inositol monophosphates (IMP) [26] and glycogensynthase kinase 3 (GSK3) [27], molecules with possible links to circadian rhythms. While the role of IMP in circadian timing is relatively unexplored, our recent work indicates inositol metabolism affects rhythms [28]. GSK3 is known to regulate the stability and turnover of clock proteins, and GSK3 inhibition has effects on circadian rhythm amplitude and period [29-32]. These changes in period may facilitate phase shifting, improve entrainment and have important implications for lithium's effects on mood. In the dopamine transporter (DAT) knockdown model of mania, mice have a longer circadian period, and the period was shortened by the mood stabilizer valproic acid [33]. Similar period shortening effects of valproic acid were observed in gene expression rhythms in cells from BD patients [33], an effect on rhythms similar to selective GSK3 inhibitors [32]. In humans, clinical interventions including partial sleep deprivation and phototherapy both advance circadian phase and facilitate the therapeutic effects of lithium [34]. These data together indicate that period shortening and/or phase advancing may have beneficial effects on mood, possibly by correcting mismatches between the environmental light/dark cycle and endogenous rhythms that occur in the context of long period and/or phase delays.

Based on the observations that circadian rhythm abnormalities are central to BD, that lithium response is genetically encoded, and that lithium affects the circadian clock, we hypothesized lithium responsive $\mathrm{BD}$ patients (Li-R) would have identifiable differences in circadian rhythms compared to lithium nonresponders (Li-NR). Moreover, we expected that we could use identifiable circadian biomarkers to better predict therapeutic outcomes after lithium pharmacotherapy.

\section{METHODS}

Lithium Clinical Trial

Clinical response to lithium was determined prospectively in between January 2011 and January 2016 through the PGBD multi-center treatment trial [35]. Additional detail is summarized in Supplementary Methods.

\section{Determination of clinical response to lithium}

Subjects with BD type I were transitioned to lithium monotherapy over 12-weeks. Subjects able to stabilize on lithium monotherapy were classified as lithium responders (Li-R) and entered maintenance treatment. Subjects who were unable to stabilize were classified as lithium non-responders (Li-NR) and discontinued from the study. Subjects who left the study for intolerable side effects or reasons unrelated to their clinical response to lithium were excluded from further analysis. After these exclusions, 193 subjects were considered $(N=135 \mathrm{Li}-\mathrm{R} / \mathrm{N}=58 \mathrm{Li}-\mathrm{NR})$. Subjects in maintenance were evaluated in person every two months, until either the occurrence of a depressive/manic episode (defined by DSM-IV criteria), or the end of the 2-year follow-up period.

\section{Chronotype analysis}

At baseline, subjects completed the Basic Language Morningness scale (BALM), a validated, 13-item self-reported measure of chronotype $[36,37]$. Higher BALM values correspond to a greater level of morningness.

Analysis of depressive and manic symptoms, and suicide history Subjects completed the 16 item Quick Inventory of Depressive Symptomatology Self-Report (QIDS-SR16) [38], the Clinician Administered Rating Scale for Mania (CARS-M) [39], and a detailed self-report of past suicidal behaviors. Scoring was conducted in accordance with the respective protocol for each scale. To minimize survival bias, only baseline mood measures were considered.

\section{Cell culture}

Fifty-nine study participants with informative clinical outcomes $(N=44 \mathrm{Li}-\mathrm{R} / 15 \mathrm{Li}-\mathrm{NR})$ donated skin biopsies for fibroblast cell lines (Supplementary Methods). To facilitate studies of molecular mechanisms, we also used a mouse NIH3T3 fibroblast line stably transfected with the Per2-luc reporter. The NIH3T3 model recapitulates key features of lithium's effects on rhythms $[28,40,41]$ and allowed us to conduct drug and transfection studies that would not be practical in primary human fibroblasts. As a fibroblast line, we reasoned that the results are likely comparable across cell types.

\section{Measurement of cellular circadian rhythms}

Chronotype is determined in part by circadian period and phase, either of which either individually or in combination affect the onset of activity and entrainment to a $24 \mathrm{~h}$ light/dark cycle. Period change is a pharmacological effect of lithium, and we have shown previously that cells from BD patients show irregular amplitude responses to lithium [19]. Therefore, we examined three circadian rhythm parameters (amplitude, period, phase) in fibroblasts under both lithium-treated and untreated conditions using fibroblasts donated by Li-R and Li-NR subjects. We hypothesized that short circadian period would predict clinical stability, and that Li-R cells, especially those with a longer period, would exhibit greater shortening of circadian period by lithium compared to Li-NR cells. To determine if lithium may reverse the long period abnormality associated with Li-NR, we examined the degree to which period changed in Li-R and Li-NR cells after treatment with lithium. Cellular rhythms were measured in parallel under lithium (1 mM) treated and untreated (baseline) conditions [19]. Data were fit to a damped sine wave and rhythm parameters were calculated using 
commercial software (Lumicycle Analysis). Time of peak signal (first peak after synchronization) was used as a phase marker.

\section{Drugs}

Because lithium non-selectively inhibits inositol monophosphate (IMP), we tested whether inhibition of IMP affects circadian rhythms. Cells originating from the same culture were treated simultaneously in parallel with active drug or vehicle control. Lithium chloride was purchased from Sigma. 2Aminoethoxydiphenylborane (APB), and [1-(4-Hydroxyphenoxy) ethylidene] bisphosphonic acid (L-690330) were purchased from Tocris Biosciences. Concentrated drug solutions were added to the growth media and diluted to the desired concentration. Drugs were dissolved in water or DMSO.

\section{Gene knockdown}

Knockdown of Itpr3 was performed using siRNA in NIH3T3 cells stably expressing Per2-luc under hygromycin selection [28]. See Supplementary Methods for details. Commercially available siRNAs (Dharmacon) were used according the manufacturer's protocol. Bmal1 (also called Arntl, M-040483-01-0005) was targeted as a positive control, and siRNA that does not target any known transcript served as negative control (D-001206-14-05).

Measurement of cellular $\mathrm{IP}_{3}$

$\mathrm{NIH} 3 \mathrm{~T} 3$ cells were treated with lithium $10 \mathrm{mM}$ or vehicle for $24-\mathrm{h}$ $\mathrm{IP}_{3}$ was measured using ELISA following the manufacturer's protocol (Life Span BioSciences).

\section{Genotyping}

Selective inhibition of GSK3 shortens circadian period [32], whereas lithium, a non-selective GSK3 inhibitor has been shown to paradoxically lengthen period in fibroblasts in a concentrationdependent manner [19]. Therefore, individual differences in the response of cellular period length to lithium may be relevant, possibly reflecting the varying balance of lithium's pharmacological effects on GSK3 vs. competing pathways. Therefore, we tested whether genetic variation in the $\mathrm{IP}_{3}$ system and GSK3B explains differences in period among Li-R/Li-NR. BD subjects were genotyped at $\sim 420 \mathrm{~K}$ single nucleotide polymorphisms (SNP) on PsychChip by TGEN (Phoenix, AZ). Data were deposited on the Genetic Cluster Computer (http://www.geneticcluster.org) for quality control, processing and analysis, using the PGC pipeline (https://github.com/nievergeltlab). Imputation was based on 1000 Genomes phase 3 (1KGP phase 3 ) using the first five ancestry informative principle components as covariates [42].

\section{Multifactorial model of lithium response}

We developed post hoc four criteria by which to score donors on cellular circadian factors associated with lithium response: (1) Period $<25.8 \mathrm{~h}$ (shorter than median). (2) Period $>25.8 \mathrm{~h}$ and corrected by lithium to $<25.8 \mathrm{~h}$. (3) Period $>25.8 \mathrm{~h}$ and $\mathrm{Tm}<0.79$ (top quartile phase advanced). (4) Rare allele ITPR3 genotype. The first three criteria were considered protective factors, while the fourth was considered a risk factor for non-response. One point was assigned for each protective factor, and one point subtracted for each risk factor (range of scores -1 to 3 ). Mean scores were compared across the two groups using a two-tailed $t$ test. Specificity and sensitivity for Li-NR were calculated based on the absence of any protective feature $($ score $<1)$.

\section{Statistical analyses}

Chronotype analysis was conducted in SPSS (version 20) using ANCOVA, corrected for age, race, and sex. Cellular analyses were conducted to examine three related hypotheses evaluating how circadian rhythms associate with treatment response: baseline period, baseline phase, and pharmacological effects of the drug on period/phase. Given our modest sample size, and that the dependent variables were not independent, a stringent correction for multiple comparisons was not employed. The cellular rhythm analyses were completed using GraphPad Prism (version 5.0) using a two-tailed $t$-test or one-way analysis of variance, and univariate regression analyses of phase and period. An F-test was incorporated into all analyses to ensure the assumption of equal sample variance was satisfied. Group differences in linear regressions were assessed for statistical significance using Fisher's $r$ to $z$ transformation. Genotypic analysis of period was conducted using linear regression under a dominant model. All analyses used $a<0.05$.

Human subjects research oversight

The research protocol was reviewed and approved by the local IRBs pertaining to each participating site to ensure compliance with all pertinent regulations regarding the ethical conduct of human subjects research. All subjects provided informed consent to participate.

\section{RESULTS}

Clinical trial subjects

193 lithium-treated subjects were considered for chronotype analysis (Table S1). The majority $(N=135)$ advanced to the maintenance treatment, while 58 failed to achieve maintenance (Li-NR). Of the 135 Li-R subjects, 104 ultimately had informative clinical outcomes after 2 years, while the remainder left the study before the 2-year endpoint for reasons unrelated to mood stability (Figure S1).

Chronotype is associated with lithium response

Chronotype in people with BD is a stable trait (Figure S2). Past research indicates that low morningness is associated with depression [16], and worse treatment outcomes in major depression [43]. Therefore, we examined chronotype to determine if it was a predictor of lithium response in BD. Using the categorical measure of lithium response, Li-R subjects were significantly higher in morningness compared to Li-NR (mean $\mathrm{BALM}=36.1$ vs. $32.5, p<0.02$, Fig. 1a). We then analyzed separately three specific sub-groups: subjects who failed to stabilize initially, relapsed during maintenance, and achieved long-term stability. The overall group difference in morningness was significant $(p<0.01)$. Post hoc tests revealed that there was no difference in chronotype between those who failed to stabilize and those who relapsed (mean BALM 32.5 vs. 34.4), but that among those who remained stable for 2 years, morningness was significantly higher compared to those who failed to stabilize (Fig. 1b, mean BALM 37.1 vs. 32.5, $p<0.05$ ).

In order to understand the particular factors driving the association between chronotype and lithium response, we analyzed specific symptoms of BD. We found that more severely depressed subjects were lower in morningness (Fig. 1c). The most strongly associated depressive features were two sleep items: excessive sleepiness and delayed sleep onset (Table S2). Other functions that typically vary rhythmically over the day like energy, concentration, and psychomotor retardation showed similarly strong negative associations. However, additional depressive symptoms that are not commonly considered rhythmic, including feelings of sadness, and low self-esteem were also negatively associated with morningness.

Similarly, patients with more manic symptoms showed significantly reduced morningness scores (Fig. 1d), and several individual manic symptoms on the CARS-M scale, showed significant negative association with BALM, indicating that decreased need for sleep, disorganization and disorientation were all greater in subjects with low morningness (Table S3).

Finally, we examined suicide attempt (SA) history and chronotype. Subjects who endorsed one or more previous SA 
A Chronotype and Lithium Response

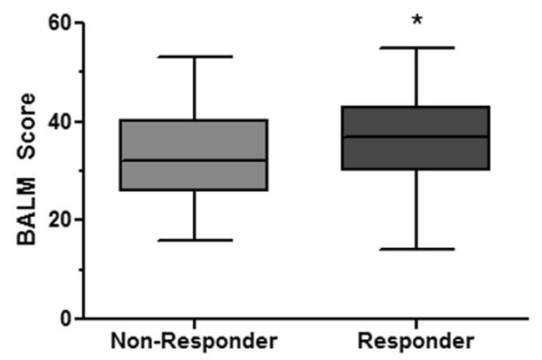

C Chronotype and Depression

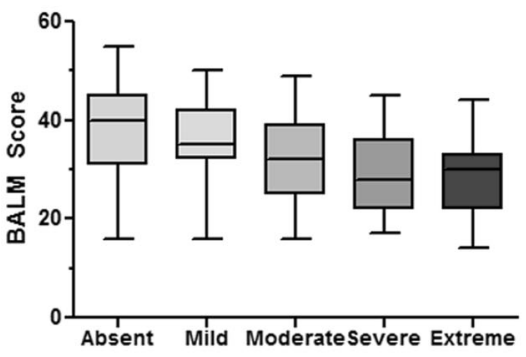

B Chronotype and Lithium Response
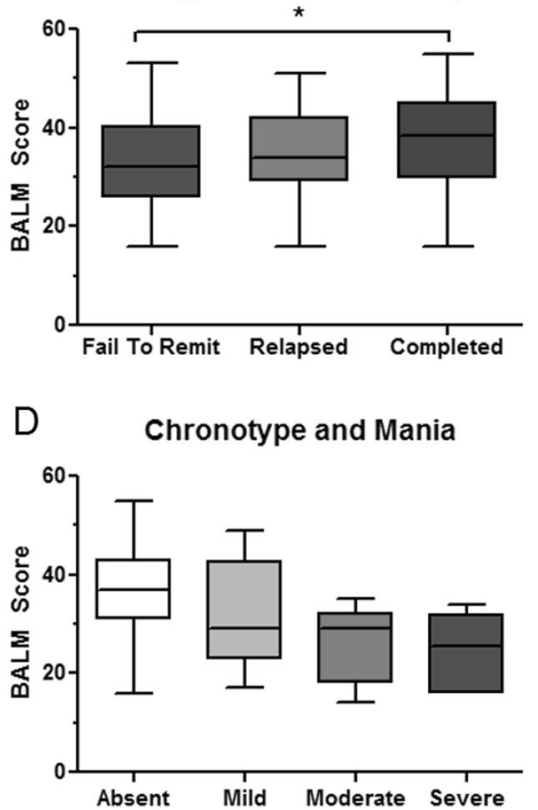

E

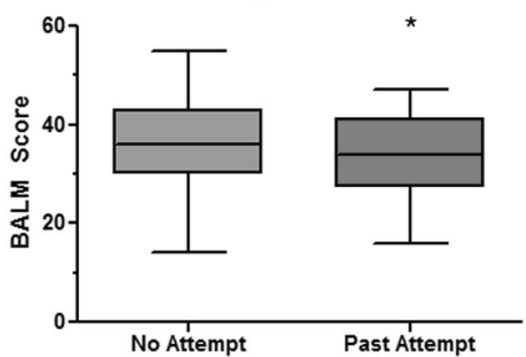

Fig. 1 Chronotype is associated with clinical response to lithium. a Li-R subjects (entered the maintenance phase, $N=135$ ) were significantly higher in trait morningness at baseline compared to Li-NR (those who failed to stabilize, $N=57$ ). $\mathbf{b}$ Those who failed to stabilize mood ( $N=58$ ) and those who suffered mood relapse during maintenance $(N=41)$ were similarly low in morningness. Morningness was highest in those who remained stable for 2-years $(N=63)$. Morningness is negatively correlated with c baseline depression (for absent $/ \mathrm{mild} / \mathrm{moderate} / \mathrm{severe} /$ extreme $N=69 / 52 / 31 / 44 / 14$ ) d baseline symptoms of mania (for absent/mild/moderate/severe $N=148 / 26 / 9 / 6$ ). e Morningness is negatively correlated with past suicide attempts ( $N=135$ no attempt / $N=54$ past attempt). Numbers vary slightly across analyses due to missing data. Box plots indicate minimum, maximum and mean values. Error bars indicate SEM. * Indicates $p<0.05$

were significantly lower in morningness compared to subjects who had not attempted suicide (Mean BALM: $\mathrm{SA}=32.9$ vs. no $\mathrm{SA}=35.9, p<0.05$, Fig. 1e).

Cellular rhythms

There were no significant demographic differences between Li-R and Li-NR cell line donors (Table S4). As expected, the duration of stability on lithium (Mean \pm SEM) was significantly different $(p<0.001)$ with $568.9 \pm 39$ days for Li-R $(N=44)$ vs. $97 \pm 9$ days for Li-NR $(N=15)$. BALM scores among the BD cell line donors reflected the same overall trends observed in the larger clinical cohort, with higher morningness scores in the Li-R group (Mean BALM Li-R 37.3 vs. Li-NR 31.9, $p=0.05$ ). BALM score was nominally correlated with cellular phase in the expected direction $(r=-0.18)$, but this phase advance was not statistically significant. BALM score was not significantly correlated with any other cellular rhythm parameter.

\section{Amplitude}

There was no significant difference in baseline amplitude in cells from Li-R and Li-NR, and no effect on amplitude from lithium, indicating that the drug treatment did not increase amplitude in the cells obtained from either BD group (Fig. 2a). This finding independently replicates our previous work showing that unlike cells from controls in which lithium typically increases amplitude by $30-40 \%$, lithium does not increase amplitude in fibroblasts from BD patients [19].

\section{Period}

There was a difference in cellular period between Li-R and Li-NR donors that trended towards significance (mean period: Li-R 25.5 \pm 0.14 vs. Li-NR $26.0 \pm 0.17 \mathrm{~h} p=0.08$, Fig. $2 \mathrm{~b}$ ). Further inspection revealed that the cellular periods were not uniformly distributed between groups. Using a median split, a majority (25/29 or $86.2 \%)$ of short period $(<25.8 \mathrm{~h})$ samples came from Li-R donors, while the majority of Li-NR cells (11/15 or $73 \%$ ) had long periods (Fig. 2c). These results indicate that the odds of non-response to lithium are significantly higher in cell donors with long period $(\mathrm{OR}=3.6, p<0.05)$. However, there was overlap between Li-R and Li-NR. The results indicate that short period may be predictive of response, and that while long period may be a relative risk factor, alone it is insufficient to explain non-response. Therefore, additional mitigating factors may influence responsiveness in subjects with long period.

\section{Phase}

There were no baseline differences in phase in cells from $\mathrm{Li}-\mathrm{R}$ and Li-NR donors, and exposing the cells to lithium had no significant effect on phase. However, the relationship between period and phase differed between cells from Li-R and Li-NR. In cells from Li-R there was an inverse linear relationship between period and phase. Li-R cells with long period were typically phase advanced. In Li-NR cells this relationship was not statistically significant. Unlike Li-R cells, Li-NR cells with a long period were no more likely to be phase advanced than cells with a short period. The difference in the variance explained by these relationships was significantly different $(z=2.01, p<0.05)$ between Li-R and Li-NR (Fig. 3a, b).

Pharmacological effects of lithium

In Li-R cells there was a positive correlation $(r=0.27, p=0.06)$ between period length and the degree to which lithium shortened period (Fig. 4a). This relationship was attenuated in the Li-NR cells ( $r=0.15, p=0.58$, Fig. 4b). To investigate further, we used a median split to sub-divide Li-R/Li-NR into long and short period sub groups. Compared to Li-R cells with a short period, Li-R cells with long period shortened period to a greater degree $(p<0.05$, Figs. 4c, d). In Li-NR cells, lithium had no effect on period length in either group. Therefore, in the subset of Li-R cells with a longer circadian period, lithium treatment favors period shortening. 
A Amplitude Change After Lithium

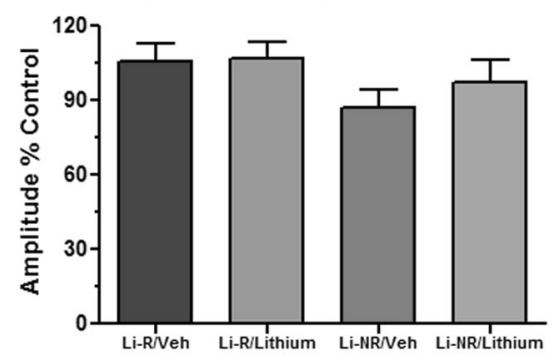

C Period and Lithium Response

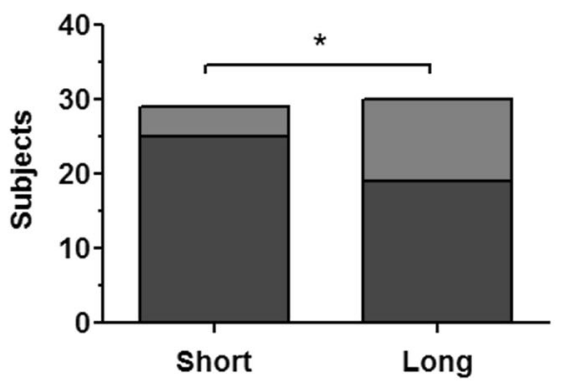

B

Period And Lithium Response

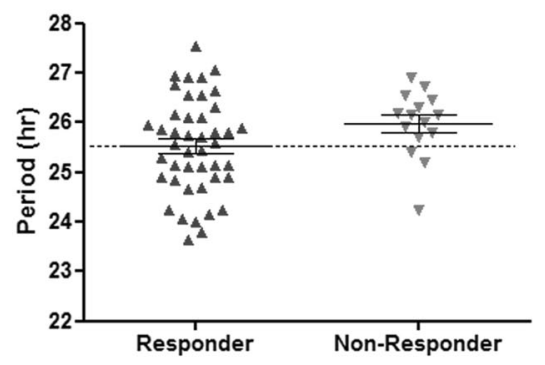

D Period and Lithium Response
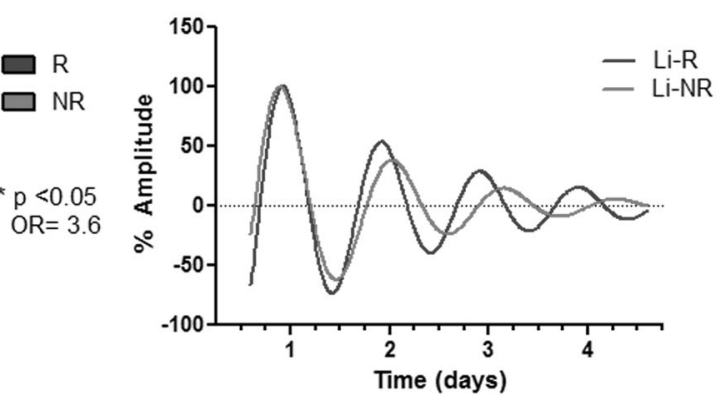

Fig. 2 Circadian rhythm parameters in cells from Li-R and Li-NR donors. a There was no difference in amplitude in cells donated by Li-R $(N=44)$ and Li-NR $(N=15)$, either at baseline (untreated) or after cells were treated with lithium $1 \mathrm{mM}$. b Period length was more likely to be short in cells from Li-R donors compared to cells from Li-NR. Mean period Li-R $25.5 \pm 0.14 \mathrm{vs}$. Li-NR $26.0 \pm 0.17 \mathrm{~h}$, two-tailed $T$ test indicates $p=0.08$. c The proportion of Li-R and Li-NR differs significantly in donors with short and long circadian period $\left[X^{2}=4.06(1)\right.$, OR $=3.6$, ${ }^{*}$ indicates $\left.p<0.05\right]$. Short/Long corresponds to below/above the median period of $25.8 \mathrm{~h}$. d Representative rhythm traces of cells from Li-R (blue) and Li-NR (red) subjects. Error bars represent SEM

A

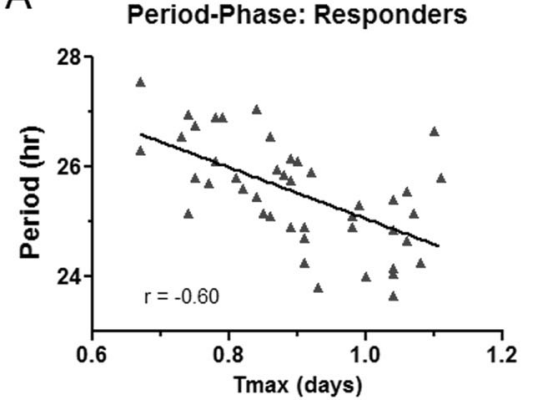

B

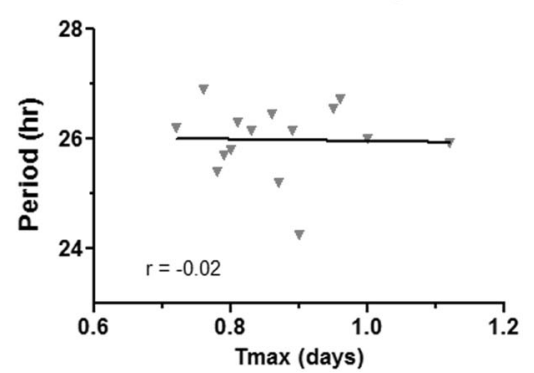

C Phase in Long Period Cells

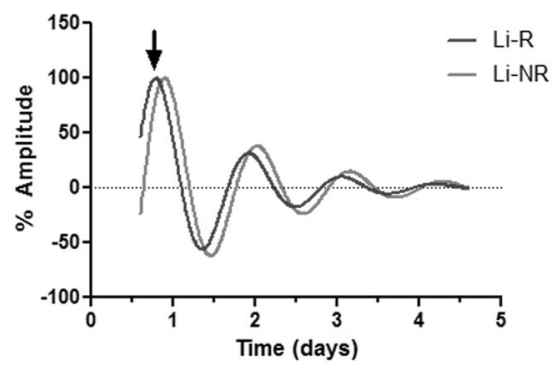

Fig. 3 The phase-period relationship differs in cells from Li-NR compared to Li-R. a In Li-R cells $(N=44)$, samples with the longest period are phase advanced relative to those with shorter periods. b In Li-NR cells $(N=15)$, there exists no significant relationship between period and phase. c Representative rhythm traces showing the relative phase advance of Li-R cells vs Li-NR cells with similarly long periods. Arrows indicate phase marker (Tmax)

Period change by lithium is affected by $\mathrm{IP}_{3}$

The selective IMP1/2 inhibitor L-690330 caused a concentrationdependent lengthening in circadian period in NIH3T3 cells (Fig. 5a, b). IMP inhibition is predicted to increase the intracellular levels of 1,4,5-trisphosphate $\left(\mathrm{IP}_{3}\right)$ by reducing its turnover. Supporting this hypothesis, we found that 24-h treatment of NIH3T3 cells with lithium $10 \mathrm{mM}$ (a concentration that lengthens period) revealed a trend towards increased $\mathrm{IP}_{3}$ levels as expected (Fig. 5c). Accordingly, we hypothesized that intracellular IP 3 receptors (IP3R) may explain some of the period lengthening effect of lithium. In line with this expectation, the IP3R antagonist APB caused a concentration-dependent period shortening and attenuated the period lengthening effect of lithium (Fig. $5 \mathrm{~d}$ ). Furthermore, genetic knockdown of $I t p 3 r$, the predominant $\mathrm{IP}_{3}$ receptor gene in NIH3T3 fibroblasts also blocked the period lengthening effect of lithium (Fig. 5e, f). Using publicly available data (https://data.broadinstitute.org $/ \mathrm{mpg} / \mathrm{ricopili),} \mathrm{we} \mathrm{determined} \mathrm{that} \mathrm{a} \mathrm{common} \mathrm{variant}$ in ITPR3 (rs11758031) showed a modest nominal association with BD $\left(p<6 \times 10^{-4}\right)$ in a previous GWAS of BD [44], while neither ITPR1 or ITPR2 showed similar trends. To test the role of ITPR3 in fibroblasts from BD patients, we examined whether this variant is associated with period. We found that when treated with lithium, cells with ITPR3 minor A alleles showed significantly longer period compared to those with two common alleles (AA/AG: $26.04 \pm 0.26$ vs. GG: $25.44 \pm 0.13$, Fig. $5 \mathrm{~g}$ ). Unlike at higher concentrations of lithium, the $-50 T / C$ variant in GSK3B ( $r 5334558$ ) was not associated with period change after lithium $1 \mathrm{mM}$ (Fig. 5h).

Modelling lithium response using circadian factors To explore the overall relationship of circadian variables to lithium response, we developed a model to predict clinical lithium response that could assess four variables in cells (baseline period, period after lithium, phase, and ITPR3 genotype). Of $40 \mathrm{Li}-\mathrm{R}$ cell line donors, $33(83 \%)$ had a score of 1.0 or more, indicating a 


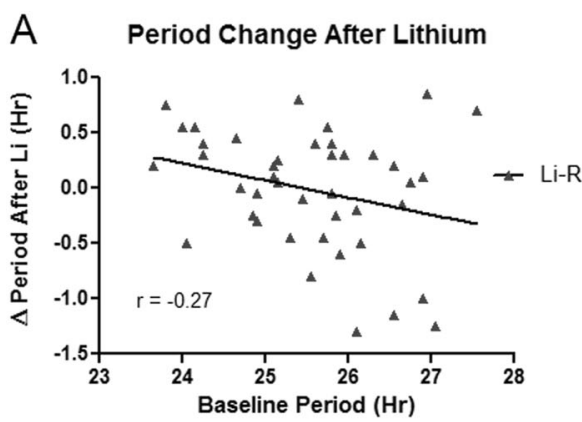

C Period Change After Lithium

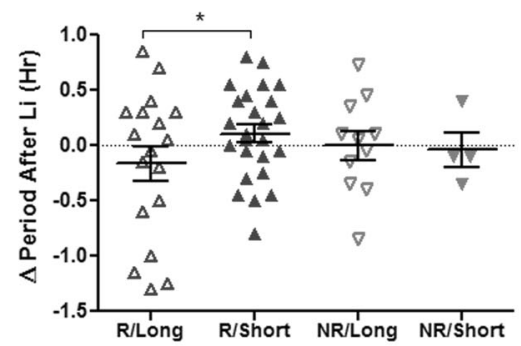

B Period Change After Lithium

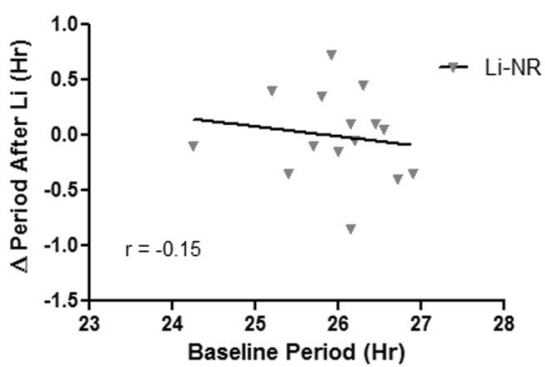

Effect of Lithium: Responder/Long Period

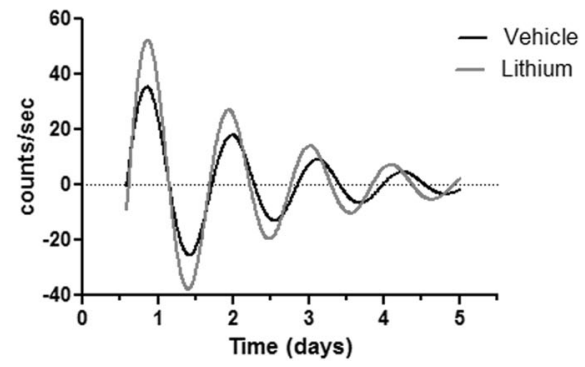

Fig. 4 Lithium shortens circadian period preferentially in cells from Li-R donors. a In Li-R subjects the period shortening effect of lithium inversely correlates with baseline period length. Li-R subjects with long $(>25.8 \mathrm{~h})$ periods show the greatest shortening effect of lithium. $\mathbf{b}$ In Li-NR subjects the relationship between baseline period length and the effect of lithium on period is attenuated and no longer significant. c) Li-R cells with long period $(N=18)$ show significant period shortening after lithium compared to Li-R cells with short period $(N=25)$. Lithium had no effect on period length in Li-NR with long $(N=11)$ or short $(N=4)$ period. ${ }^{*}$ Indicates $p<0.05$. d Representative rhythm traces of a Li-R cell with long period after treatment with vehicle or lithium $1 \mathrm{mM}$

preponderance of protective factors. Among Li-R only one cell line ( $3 \%$ of $\mathrm{Li}-\mathrm{R})$ had more than one protective factor, indicating minimal overlap among factors. The mean $( \pm S E M)$ composite score among Li-R was $0.85 \pm 0.15$. Among $15 \mathrm{Li}-\mathrm{NR}$ cell line donors, $12(80 \%)$ scored $\leq 0$, indicating a preponderance of risk factors and the mean score was $0.07 \pm 0.07$ (Figure S3). Accordingly, our four-factor model had a specificity of $83 \%$ and sensitivity of $80 \%$ for identifying Li-NR.

\section{DISCUSSION}

We have shown that chronotype and cellular circadian rhythms predict lithium response in BD. Higher morningness was associated with fewer depressive and manic symptoms, fewer SAs and longer duration of mood stability. In cells, short circadian period predicted lithium response in the donor. In cells with a long period, pharmacologically induced period shortening by lithium or phase advance was also associated with favorable clinical response. Finally, we identified IMP inhibition as a possible mechanism by which lithium affects circadian period. Taken together, our data support the hypothesis that features of circadian period and phase predicted to advance rhythms may positively contribute to lithium responsiveness. The results suggest a model by which lithium response could be predicted using circadian factors. It remains an open question whether in the course of lithium treatment circadian factors impact directly upon pathophysiological mechanisms causally related to BD, or non-specifically modify symptom burden.

Most previous studies of lithium response utilized retrospective clinical assessment and subjects treated with multiple psychotropic medications. A strength of our study is that our relatively large cohort of BD subjects was evaluated prospectively on a standardized lithium monotherapy protocol and that cells were available from these well-characterized donors. Our study also has limitations. We did not have cell lines from every clinical trial subject and accordingly, the sample available for cellular analyses, while comparatively large for this kind of study, is incomplete and had relatively few Li-NR lines. In addition, our cellular model of the 24-h circadian cycle has caveats. Estimates of circadian parameters calculated in vitro likely differ from those obtained in vivo due to artifacts associated with the cell culture environment and the use of peripheral cells. Moreover, with particular respect to phase, the reference point in vitro is the synchronizing biochemical stimuli associated with medium change, whereas in vivo, phase reference point is determined by light exposure. While our chronotype findings in BD patients largely support our cell-based conclusions, underlying mechanistic differences in phase setting could limit their overall generalizability in vivo. Finally, without an independent cohort, we were unable to validate our predictive model in an independent data set.

We reported previously that compared to controls, cells from BD patients had longer circadian periods and were more likely to show a period lengthening effect from lithium at a therapeutically relevant concentration, especially in cells from BD patients with a history of SA [19]. These previous studies were conducted in cells collected from hospitalized patients with complex medication regimens. Therefore, it was previously impossible to determine the relationship between circadian rhythms and lithium response. The present study extends this previous work, and demonstrates that period modulation by lithium may have relevance not only as a discriminator of $B D$ from control cells, but also for predicting therapeutic outcomes to lithium monotherapy. Our previous work also found that lithium amplifies circadian rhythms in control cells, but not in BD cells $[19,40,41]$. In this study, we found that the attenuation of this circadian amplitude phenotype in BD was present regardless of $\mathrm{Li}-\mathrm{R} / \mathrm{Li}-\mathrm{NR}$ status. Therefore, it is a phenotypic marker of $\mathrm{BD}$ that may point to molecular mechanisms underlying the 


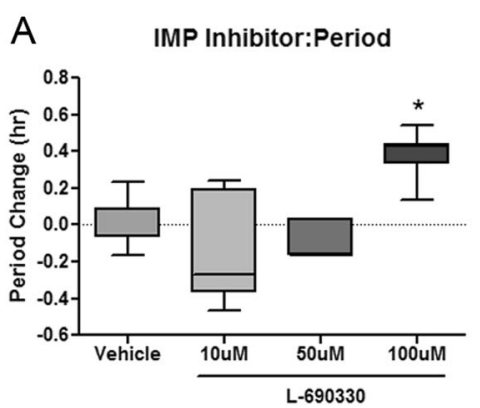

D Li + IP3R Antagonist: Period

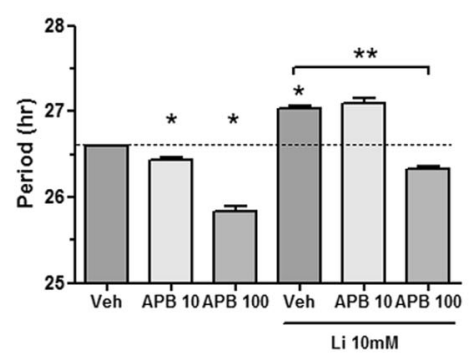

G

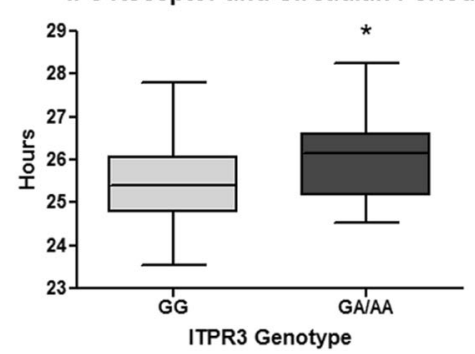

B

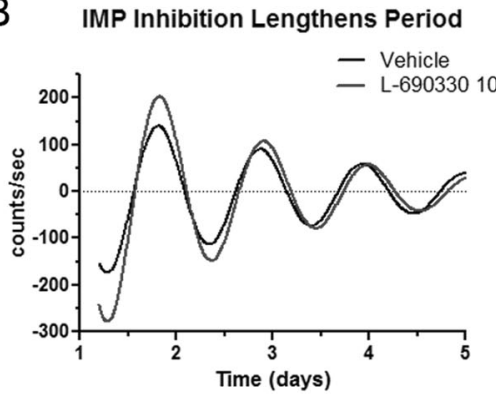

E

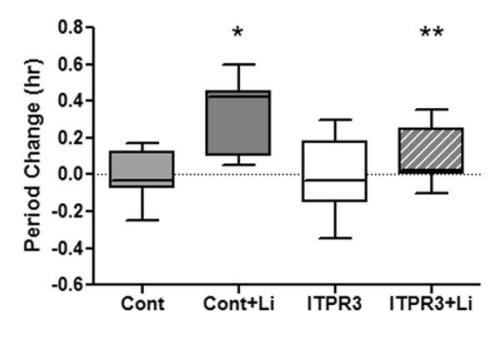

H GsK3B and Circadian Period

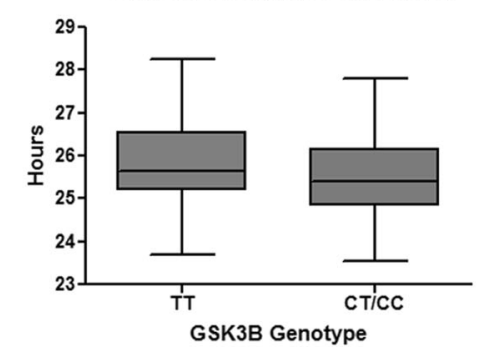

C Lithium Increases $\mathbb{P}_{3}$

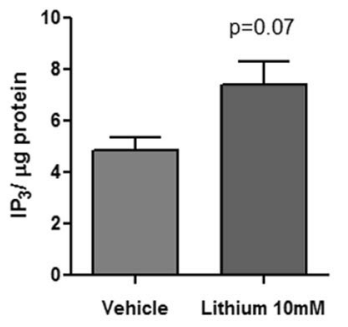

$\mathrm{F}$

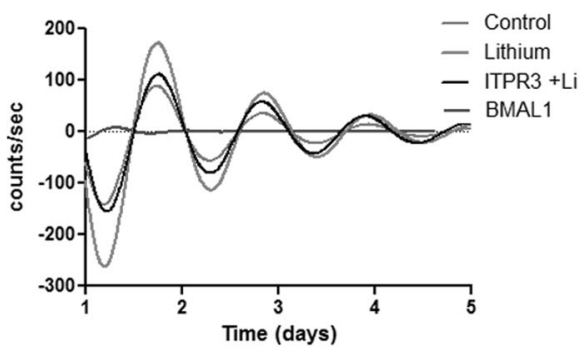

Fig. 5 Inhibition of IMP causes period lengthening. Despite its action as an inhibitor of GSK3, lithium lengthens circadian period at high concentrations, perhaps indicating circadian effects of IMP inhibition. a Selective inhibition of IMP with L-690330 causes period lengthening $(N=6-9 /$ group). b Representative trace of the period lengthening effect of L-690330. c Treatment of mouse fibroblast cell lines with lithium for $24 \mathrm{~h}$ caused a nominal increase in $\mathrm{IP}_{3}$ that trended towards statistical significance $(N=7-8 / \mathrm{group})$. $\mathbf{d}$ Antagonism of the $I \mathrm{P}_{3}$ receptor shortens period and reverses the period lengthening effects of lithium $\left(N=3 /\right.$ group). e Knockdown of the IP $\mathrm{P}_{3}$ receptor gene Itpr3 in NIH3T3 cells $(N=8-14 /$ group) blocks the period lengthening effect of lithium (10 mM). f Representative traces of Itpr3 knockdown experiment. Itpr3 knockdown attenuates the period lengthening effect of lithium. The loss of rhythm from Bmal1 knockdown confirms efficient siRNA transfection. $\mathbf{g}$ In cells from BD patients, ITPR3 genotype at the variant rs 11758031 predicts circadian period after treatment of the cells with lithium ( $N=48 \mathrm{GG}$ and $14 \mathrm{GA} / \mathrm{AA}, p<0.01)$, whereas $\mathbf{h}$ GSK3B genotype at rs334558 (-50T/C) does not $(N=23 \mathrm{TT}, 38 \mathrm{CT} / \mathrm{CC}, p=0.65)$. Box plots indicate minimum, maximum and mean values. Error bars represent SEM. * Indicates $p<0.05$ vs control. ${ }^{* *}$ Indicates $p<0.05$ vs lithium treated

illness, but is not of any apparent prognostic value for predicting lithium response.

Mood implications for circadian misalignment

Circadian rhythms entrain to environmental cues, most notably light. The intrinsic human circadian oscillator runs with a period of $24.2 \mathrm{~h}$ [45]. Therefore, there is typically a slight mismatch between the period of the endogenous circadian rhythm and the $24 \mathrm{~h}$ light/ dark cycle. Phase advancing in response to light allows the circadian clock to overcome this mismatch and entrain to a 24-h cycle. However, with large mismatches between the light/dark cycle and the endogenous rhythm, entrainment is incomplete and results in syndromes such as advanced sleep phase [17] and delayed sleep phase [18] disorders. With extreme mismatches, the failure to entrain is compete and the endogenous rhythm "free runs" independently of the light/dark cycle. Healthy subjects maintained in a laboratory under free running conditions on a $28 \mathrm{~h}$ light/dark cycle demonstrate signs of severe physiological stress, including impairments in autonomic function, cortisol, sleep, cognition, and mood [46, 47]. Therefore, misalignment of the circadian clock causes physiological stress and negatively impacts mood. While subjects living in typical environmental conditions are unlikely to encounter such large clock/light mismatches, BD subjects do have a longer period [19], and frequently show behavior patterns that undermine efficient circadian entrainment, such as less daytime activity and limited exposure to light. In this way, BD subjects may experience mild clock/light mismatches over extended periods of time [5]. Therefore, we propose that the combination of longer period and compromised circadian entrainment causes some BD subjects to experience physiological distress, altered sleep and/or disturbed affect regulation. In this context, the degree to which lithium facilitates or opposes entrainment may affect the clinical course of lithium treatment.

Inositol monophosphatase and circadian rhythms Lithium differs from selective GSK3 inhibitors with respect to its circadian effects, causing period lengthening rather than 
shortening. IMP inhibition by lithium and subsequent accumulation of intracellular $\mathrm{IP}_{3}$ may underlie this effect, and variation in ITPR3 may affect the degree to which lithium lengthens period. Therefore, the degree to which mood stability is maintained in $\mathrm{BD}$ may relate to genetic variation in the $\mathrm{IP}_{3}$ pathway. In particular, lithium response may be enhanced in individuals where the balance of lithium's effects is shifted toward GSK3 inhibition (resulting in short period) and away from IMP inhibition (resulting in long period). The rs11758031 A/G intronic nucleotide variant may be an example of a genetic factor that influences the circadian effects of lithium. The SNP has been shown to be functional, affecting the expression of ITPR3 and IP6K3, an adjacent gene involved in signal transduction (https://www.gtexportal.org/home). In a recent GWAS of sleep phenotypes in $>1$ million subjects, ITPR3 was implicated in insomnia both by gene-based analysis and exon sequencing [48]. In contrast, the most recent GWAS for BD show little evidence of association with ITPR3 [49]. Therefore, it may be that lithium's effects on $\mathrm{IP}_{3}$ relate most directly to circadian factors and sleep, and perhaps less so to BD susceptibility factors. We have previously shown that IPMK, a gene involved in both GSK3 and inositol signaling may also influence the period lengthening effect of lithium [28]. For these reasons, further work on the inositol pathway as it relates to lithium is warranted.

The role of the clock in mood stability

Presently, we cannot determine whether the circadian influence on lithium response is essential to promoting mood stability. By period lengthening, lithium could cause circadian misalignment, leading to a state of social, environmental and/or physiological distress, leaving only subjects who remain entrained to benefit from lithium's therapeutic benefits. In these ways, the circadian influence on lithium response may be mediated by a lack of circadian "side effects" and be permissive rather than causal. Alternatively, the clock genes may be causally involved, preferentially activating neuroprotective processes in $\mathrm{Li}-\mathrm{R}$ BD patients. Clock proteins like PER2, BMAL1 and REV-ERBa regulate neurogenesis [50, 51], and neuroprotective molecules involved in lithium's therapeutic mechanism such as BDNF are rhythmically expressed [52-55]. Therefore, the clock may alter lithium's pharmacological actions directly through neurogenesis and/or neuroprotection, suggesting a direct role for clock genes in modifying lithium response.

\section{CONCLUSIONS}

Circadian period and phase predict lithium response. Future experiments are needed to determine whether circadian clocks are essential for lithium response and if facilitation of entrainment is the ultimate circadian mechanism.

\section{FUNDING AND DISCLOSURE}

The authors thank the patient participants without whom this work would not be possible. The PGBD Trial was supported by NIMH/NIGMS (MH92758), The Western Norway Regional Health Authority and the Canadian Institutes of Health Research (\#64410). Secondary clinical, genetic and cell-based analyses conducted by MJM were supported by Department of Veterans Affairs (BX003431). KEB: Advisory board member Dainippon Sumitomo Pharmaceutical and Takeda Lundbeck. KG: Speakers bureau of Sunovion. JIN: Assurex Investigator; Janssen-consultant/Investigator. JRK: Pathway Genomics Investigator. The remaining authors declare no competing interests.

\section{ADDITIONAL INFORMATION}

Supplementary Information accompanies this paper at (https://doi.org/10.1038/ s41386-018-0273-8)
Publisher's note: Springer Nature remains neutral with regard to jurisdictional claims in published maps and institutional affiliations.

\section{REFERENCES}

1. Baldessarini RJ, Tondo L, Hennen J. Lithium treatment and suicide risk in major affective disorders: update and new findings. J Clin Psychiatry. 2003;64:44-52.

2. Merikangas KR, Jin R, He JP, Kessler RC, Lee S, Sampson NA, et al. Prevalence and correlates of bipolar spectrum disorder in the world mental health survey initiative. Arch Gen Psychiatry. 2011;68:241-51. https://doi.org/10.1001/ archgenpsychiatry.2011.12.

3. McKenna BS, Drummond SP, Eyler LT. Associations between circadian activity rhythms and functional brain abnormalities among euthymic bipolar patients: a preliminary study. J Affect Disord. 2014;164:101-6. https://doi.org/10.1016/j. jad.2014.04.034.

4. Jones SH, Hare DJ, Evershed K. Actigraphic assessment of circadian activity and sleep patterns in bipolar disorder. Bipolar Disord. 2005;7:176-86. https://doi.org/ 10.1111/j.1399-5618.2005.00187.x.

5. Pagani L, St Clair PA, Teshiba TM, Service SK, Fears SC, Araya C, et al. Genetic contributions to circadian activity rhythm and sleep pattern phenotypes in pedigrees segregating for severe bipolar disorder. Proc Natl Acad Sci USA. 2016;113:E754-61. https://doi.org/10.1073/pnas.1513525113.

6. Gonzalez R, Tamminga CA, Tohen M, Suppes $T$. The relationship between affective state and the rhythmicity of activity in bipolar disorder. J Clin Psychiatry. 2014;75:e317-22. https://doi.org/10.4088/JCP.13m08506.

7. Harvey AG. Sleep and circadian rhythms in bipolar disorder: seeking synchrony, harmony, and regulation. Am J Psychiatry. 2008;165:820-9. https://doi.org/ 10.1176/appi.ajp.2008.08010098.

8. Souetre E, Salvati E, Wehr TA, Sack DA, Krebs B, Darcourt G. Twenty-four-hour profiles of body temperature and plasma TSH in bipolar patients during depression and during remission and in normal control subjects. Am J Psychiatry. 1988;145:1133-7. https://doi.org/10.1176/ajp.145.9.1133.

9. Moon JH, Cho CH, Son GH, Geum D, Chung S, Kim H, et al. Advanced Circadian Phase in Mania and Delayed Circadian Phase in Mixed Mania and Depression Returned to Normal after Treatment of Bipolar Disorder. EBioMedicine. 2016;11:285-95. https://doi.org/10.1016/j.ebiom.2016.08.019.

10. Robillard R, Naismith SL, Rogers NL, Scott EM, Ip TK, Hermens DF, et al. Sleep-wake cycle and melatonin rhythms in adolescents and young adults with mood disorders: comparison of unipolar and bipolar phenotypes. Eur Psychiatry. 2013;28:412-6. https://doi.org/10.1016/j.eurpsy.2013.04.001.

11. Partch $\mathrm{CL}$, Green $\mathrm{CB}$, Takahashi JS. Molecular architecture of the mammalian circadian clock. Trends Cell Biol. 2014;24:90-9. https://doi.org/10.1016/j. tcb.2013.07.002

12. Roybal K, Theobold D, Graham A, DiNieri JA, Russo SJ, Krishnan V, et al. Mania-like behavior induced by disruption of CLOCK. Proc Natl Acad Sci USA. 2007;104:6406-11. https://doi.org/10.1073/pnas.0609625104.

13. Landgraf D, Long JE, Proulx CD, Barandas R, Malinow R, Welsh DK. Genetic disruption of circadian rhythms in the suprachiasmatic nucleus causes helplessness, behavioral despair, and anxiety-like behavior in mice. Biol Psychiatry. 2016;80:827-35. https://doi.org/10.1016/j.biopsych.2016.03.1050.

14. Schnell A, Sandrelli F, Ranc V, Ripperger JA, Brai E, Alberi L, et al. Mice lacking circadian clock components display different mood-related behaviors and do not respond uniformly to chronic lithium treatment. Chronobiol Int. 2015;32:1075-89. https://doi.org/10.3109/07420528.2015.1062024.

15. Li JZ, Bunney BG, Meng F, Hagenauer MH, Walsh DM, Vawter MP, et al. Circadian patterns of gene expression in the human brain and disruption in major depressive disorder. Proc Natl Acad Sci USA. 2013;110:9950-5. https://doi.org/ 10.1073/pnas.1305814110.

16. Lane JM, Vlasac I, Anderson SG, Kyle SD, Dixon WG, Bechtold DA, et al. Genomewide association analysis identifies novel loci for chronotype in 100,420 individuals from the UK Biobank. Nat Commun. 2016;7:10889. https://doi.org/10.1038/ ncomms10889.

17. Toh $\mathrm{KL}$, Jones $\mathrm{CR}$, He $\mathrm{Y}$, Eide EJ, Hinz WA, Virshup DM, et al. An hPer2 phosphorylation site mutation in familial advanced sleep phase syndrome. Science. 2001;291:1040-3.

18. Patke A, Murphy PJ, Onat OE, Krieger AC, Ozcelik T, Campbell SS, et al. Mutation of the human circadian clock gene CRY1 in familial delayed sleep phase disorder. Cell. 2017;169:203-15 e13. https://doi.org/10.1016/j.cell.2017.03.027.

19. McCarthy MJ, Wei H, Marnoy Z, Darvish RM, McPhie DL, Cohen BM, et al. Genetic and clinical factors predict lithium's effects on PER2 gene expression rhythms in cells from bipolar disorder patients. Transl Psychiatry. 2013;3:e318. https://doi. org/10.1038/tp.2013.90.

20. Perlis RH, Ostacher MJ, Patel JK, Marangell LB, Zhang H, Wisniewski SR, et al. Predictors of recurrence in bipolar disorder: primary outcomes from the 
Systematic Treatment Enhancement Program for Bipolar Disorder (STEP-BD). Am J Psychiatry. 2006;163:217-24. https://doi.org/10.1176/appi.ajp.163.2.217.

21. Licht RW, Vestergaard P, Rasmussen NA, Jepsen K, Brodersen A, Hansen PE. A lithium clinic for bipolar patients: 2-year outcome of the first 148 patients. Acta Psychiatr Scand. 2001;104:387-90.

22. McGuffin P, Rijsdijk F, Andrew M, Sham P, Katz R, Cardno A. The heritability of bipolar affective disorder and the genetic relationship to unipolar depression. Arch Gen Psychiatry. 2003;60:497-502. https://doi.org/10.1001/archpsyc.60.5.497.

23. Grof P, Duffy A, Cavazzoni P, Grof E, Garnham J, MacDougall M, et al. Is response to prophylactic lithium a familial trait? J Clin Psychiatry. 2002;63:942-7.

24. Song J, Bergen SE, Di Florio A, Karlsson R, Charney A, Ruderfer DM, et al. Genome-wide association study identifies SESTD1 as a novel risk gene for lithium-responsive bipolar disorder. Mol Psychiatry. 2015. https://doi.org/ 10.1038/mp.2015.165.

25. Hou L, Heilbronner U, Degenhardt F, Adli M, Akiyama K, Akula N, et al. Genetic variants associated with response to lithium treatment in bipolar disorder: a genome-wide association study. Lancet. 2016. https://doi.org/10.1016/S01406736(16)00143-4.

26. Berridge MJ, Downes CP, Hanley MR. Neural and developmental actions of lithium: a unifying hypothesis. Cell. 1989;59:411-9.

27. Klein PS, Melton DA. A molecular mechanism for the effect of lithium on development. Proc Natl Acad Sci USA. 1996;93:8455-9.

28. Wei H, Landgraf D, Wang G, McCarthy MJ. Inositol polyphosphates contribute to cellular circadian rhythms: Implications for understanding lithium's molecular mechanism. Cell Signal. 2018;44:82-91. https://doi.org/10.1016/j. cellsig.2018.01.001.

29. Harada Y, Sakai M, Kurabayashi N, Hirota T, Fukada Y. Ser-557-phosphorylated mCRY2 is degraded upon synergistic phosphorylation by glycogen synthase kinase-3 beta. J Biol Chem. 2005;280:31714-21. https://doi.org/10.1074/jbc. M506225200.

30. litaka C, Miyazaki K, Akaike T, Ishida N. A role for glycogen synthase kinase-3beta in the mammalian circadian clock. J Biol Chem. 2005;280:29397-402. https://doi. org/10.1074/jbc.M503526200.

31. Yin L, Wang J, Klein PS, Lazar MA. Nuclear receptor Rev-erbalpha is a critical lithium-sensitive component of the circadian clock. Science. 2006;311:1002-5. https://doi.org/10.1126/science.1121613.

32. Hirota T, Lewis WG, Liu AC, Lee JW, Schultz PG, Kay SA. A chemical biology approach reveals period shortening of the mammalian circadian clock by specific inhibition of GSK-3beta. Proc Natl Acad Sci USA. 2008;105:20746-51. https://doi. org/10.1073/pnas.0811410106.

33. Landgraf D, Joiner WJ, McCarthy MJ, Kiessling S, Barandas R, Young JW, et al. The mood stabilizer valproic acid opposes the effects of dopamine on circadian rhythms. Neuropharmacology. 2016;107:262-70. https://doi.org/ 10.1016/j.neuropharm.2016.03.047.

34. Wu JC, Kelsoe JR, Schachat C, Bunney BG, DeModena A, Golshan S, et al. Rapid and sustained antidepressant response with sleep deprivation and chronotherapy in bipolar disorder. Biol Psychiatry. 2009;66:298-301. https://doi. org/10.1016/j.biopsych.2009.02.018.

35. Oedegaard KJ, Alda M, Anand A, Andreassen OA, Balaraman Y, Berrettini WH, et al. The Pharmacogenomics of Bipolar Disorder study (PGBD): identification of genes for lithium response in a prospective sample. BMC Psychiatry. 2016;16:129. https://doi.org/10.1186/s12888-016-0732-x.

36. Brown FM. Psychometric equivalence of an improved Basic Language Morningness (BALM) scale using industrial population within comparisons. Ergonomics. 1993;36:191-7. https://doi.org/10.1080/00140139308967872.

37. Rhee MK, Lee HJ, Rex KM, Kripke DF. Evaluation of two circadian rhythm questionnaires for screening for the delayed sleep phase disorder. Psychiatry Investig. 2012;9:236-44. https://doi.org/10.4306/pi.2012.9.3.236.

38. Rush AJ, Trivedi MH, Ibrahim HM, Carmody TJ, Arnow B, Klein DN, et al. The 16-Item Quick Inventory of Depressive Symptomatology (QIDS), clinician rating (QIDS-C), and self-report (QIDS-SR): a psychometric evaluation in patients with chronic major depression. Biol Psychiatry. 2003;54:573-83.

39. Altman EG, Hedeker DR, Janicak PG, Peterson JL, Davis JM. The ClinicianAdministered Rating Scale for Mania (CARS-M): development, reliability, and validity. Biol Psychiatry. 1994;36:124-34.

40. McCarthy MJ, LeRoux M, Wei H, Beesley $S$, Kelsoe JR, Welsh DK. Calcium channel genes associated with bipolar disorder modulate lithium's amplification of circadian rhythms. Neuropharmacology.2015. https://doi.org/10.1016/j. neuropharm.2015.10.017.

41. McCarthy MJ, Wei H, Landgraf D, Le Roux MJ, Welsh DK. Disinhibition of the extracellular-signal-regulated kinase restores the amplification of circadian rhythms by lithium in cells from bipolar disorder patients. Eur Neuropsychopharmacol. 2016;26:1310-9. https://doi.org/10.1016/j.euroneuro.2016.05.003.

42. Nievergelt CM, Maihofer AX, Shekhtman T, Libiger O, Wang X, Kidd KK, et al. Inference of human continental origin and admixture proportions using a highly discriminative ancestry informative 41-SNP panel. Investig Genet. 2013;4:13. https://doi.org/10.1186/2041-2223-4-13.

43. Chan JW, Lam SP, Li SX, Yu MW, Chan NY, Zhang J, et al. Eveningness and insomnia: independent risk factors of nonremission in major depressive disorder. Sleep. 2014;37:911-7. https://doi.org/10.5665/sleep.3658.

44. PGC-BD. Large-scale genome-wide association analysis of bipolar disorder identifies a new susceptibility locus near ODZ4. Nat Genet. 2011;43:977-83. https://doi.org/10.1038/ng.943.

45. Czeisler CA, Duffy JF, Shanahan TL, Brown EN, Mitchell JF, Rimmer DW, et al. Stability, precision, and near-24-hour period of the human circadian pacemaker. Science . 1999:284:2177-81.

46. Scheer FA, Hilton MF, Mantzoros CS, Shea SA. Adverse metabolic and cardiovascular consequences of circadian misalignment. Proc Natl Acad Sci USA. 2009;106:4453-8. https://doi.org/10.1073/pnas.0808180106

47. Santhi N, Lazar AS, McCabe PJ, Lo JC, Groeger JA, Dijk DJ. Sex differences in the circadian regulation of sleep and waking cognition in humans. Proc Natl Acad Sci USA. 2016;113:E2730-9. https://doi.org/10.1073/pnas.1521637113.

48. Jansen PR, Watanabe K, Stringer S, Skene N, Bryois J, Hammerschlag AR, et al. Genome-wide analysis of insomnia $(\mathrm{N}=1,331,010)$ identifies novel loci and functional pathways. bioRxiv. 2018. https://doi.org/10.1101/214973.

49. Stahl E, Breen G, Forstner A, McQuillin A, Ripke S, Cichon S, et al. Genomewide association study identifies 30 loci associated with bipolar disorder. bioRxiv. 2018. https://doi.org/10.1101/173062.

50. Bouchard-Cannon P, Mendoza-Viveros L, Yuen A, Kaern M, Cheng HY. The circadian molecular clock regulates adult hippocampal neurogenesis by controlling the timing of cell-cycle entry and exit. Cell Rep. 2013;5:961-73. https://doi.org/ 10.1016/j.celrep.2013.10.037

51. Schnell A, Chappuis S, Schmutz I, Brai E, Ripperger JA, Schaad O, et al. The nuclear receptor REV-ERBalpha regulates Fabp7 and modulates adult hippocampal neurogenesis. PLoS ONE. 2014;9:e99883. https://doi.org/10.1371/ journal.pone.0099883.

52. Angelucci $F$, Aloe $L$, Jimenez-Vasquez $P$, Mathe AA. Lithium treatment alters brain concentrations of nerve growth factor, brain-derived neurotrophic factor and glial cell line-derived neurotrophic factor in a rat model of depression. Int J Neuropsychopharmacol. 2003;6:225-31. https://doi.org/10.1017/ S1461145703003468.

53. Begliuomini S, Lenzi E, Ninni F, Casarosa E, Merlini S, Pluchino N, et al. Plasma brain-derived neurotrophic factor daily variations in men: correlation with cortisol circadian rhythm. J Endocrinol. 2008;197:429-35. https://doi.org/10.1677/JOE-070376.

54. Berchtold NC, Oliff HS, Isackson P, Cotman CW. Hippocampal BDNF mRNA shows a diurnal regulation, primarily in the exon III transcript. Brain Res Mol Brain Res. 1999;71:11-22.

55. Liang FQ, Walline R, Earnest DJ. Circadian rhythm of brain-derived neurotrophic factor in the rat suprachiasmatic nucleus. Neurosci Lett. 1998;242:89-92. 